\title{
Expression of CHODL in hepatocellular carcinoma affects invasion and migration of liver cancer cells
}

\author{
ZEJIAN HUANG ${ }^{1}$, NING ZHANG ${ }^{2}$, WENDA LI $^{1}$, JUN CAO $^{1}$, LEI ZHANG $^{1}$ and YAJIN CHEN ${ }^{1}$ \\ ${ }^{1}$ Department of Hepatobiliary and Pancreatic Surgery, Sun Yat-Sen Memorial Hospital, \\ Sun Yat-Sen University, Guangzhou, Guangdong 510120; ${ }^{2}$ Department of Gastroenterology and Hepatology, \\ The First Affiliated Hospital of Sun Yat-Sen University, Guangzhou, Guangdong 510000, P.R. China
}

Received July 3, 2015; Accepted September 30, 2016

DOI: $10.3892 / \mathrm{ol} .2016 .5466$

\begin{abstract}
Hepatocellular carcinoma (HCC) is the third leading cause of cancer-associated death. Due to rapid progression and metastasis, the long-term survival remains poor for most patients. Thus, it is important to discover and develop novel preventive strategies and therapeutic approaches for HCC. Recent data show that chondrolectin (CHODL) is commonly overexpressed in the majority of lung cancers, indicating a possible correlation between CHODL and metastasis of lung cancer cells. Our investigation shows that the expression of CHODL is significantly decreased in HCC clinical samples and in HCC cell lines. Overexpression of CHODL in SMMC7721 cells with a lentiviral vector increased SMMC7721 cell migration and invasion. Our findings establish for the first time an association between human CHODL and HCC metastasis.
\end{abstract}

\section{Introduction}

Hepatocellular carcinoma (HCC) represents more than $95 \%$ of primary liver cancers, with more than half a million new cases annually $(1,2)$, ranking as the fifth most common malignant tumor worldwide and the third leading cause of cancer-associated death $(1,3)$. Due to the asymptomatic development at early stages, deficiencies in early diagnostic accuracy, rapid progression and metastasis, limited treatment options, and frequent postoperative recurrence, HCC displays a dismal prognosis, coupled with high mortality, and has become a major public health problem, particularly in developing countries (3-5).

Patients who are diagnosed early can often be cured by surgical resection, liver transplantation or radiofrequency ablation, leading to an increase in overall survival (4). However, for most HCC patients, therapy remains difficult due to a high

Correspondence to: Dr Yajin Chen, Department of Hepatobiliary and Pancreatic Surgery, Sun Yat-Sen Memorial Hospital, Sun Yat-Sen University, 107 Yanjiang Road West, Guangzhou, Guangdong 510120, P.R. China

E-mail: chenyajin0630@163.com

Key words: CHODL, hepatocellular carcinoma, migration, invasion frequency of relapse after resection or liver transplantation (1). In HCC with extrahepatic spread, only an anti-angiogenic and mitogen-activated protein kinase inhibitor, sorafenib, has been shown to improve overall survival of patients from 8 to 11 months $(1,2,5)$. For advanced HCC patients, intraarterial chemoembolization or percutaneous ethanol injection lead to minimal survival benefits $(6,7)$. Therefore, it is of distinct importance to discover and develop novel preventive strategies and therapeutic approaches for HCC.

The human chondrolectin (CHODL) gene, located on chromosome 21q21, is detected as a $2.6-\mathrm{kb}$ transcript containing six exons and five introns (8). The open reading frame of CHODL encodes a type I transmembrane $\mathrm{N}$-glycosylated protein consisting of 273 amino acids (molecular weight, $236 \mathrm{kDa}$ ), containing a single carbohydrate recognition domain (CRD) for C-type lectins in the extracellular region $(8,9)$. CHODL shows predominantly perinuclear localization in transiently transfected COS1 cells, and immunofluorescent staining also reveals strong punctate signals in the cytosol and cell membrane (8). Reverse transcription-polymerase chain reaction analysis and immunohistochemistry (IHC) images demonstrate that the expression of CHODL is mainly limited to vascular muscle of the testis, smooth muscle of the prostate stroma, heart muscle, skeletal muscle, crypts of the small intestines and red pulp of the spleen $(8,9)$.

In general, type I transmembrane proteins containing a C-type lectin CRD motif display a wide variety of functions, including cell recognition, complement activation, embryonic development and immune regulation (10). In mice, CHODL expression is tightly regulated during early embryonic development, which is of great importance for interactions between growth cones of motor axons and the horizontal myoseptum $(11,12)$. CHODL aberrant expression is demonstrated in spinal muscular atrophy (SMA) mouse models $(11,12)$. CHODL is highly expressed in motor neurons and has distinct effects on neurite outgrowth in zebrafish (11). CHODL messenger (m) RNA expression in human adults was found predominantly in muscle cells, suggesting a basic role in muscle formation (8). The CHODL splice isoforms (CHODLf, CHODL $\delta E$ and CHODLf $\delta E$ ) of the CHODL family were found to be differentially expressed in thymocytes and lymphocytes, indicating an association with human $\mathrm{T}$ cell development and maturation $(10,13)$. 
CHODL may be involved in tumor metastasis processes, including cell recognition, communication, cell-cell adhesion or interactions with the extracellular matrix (ECM) (14). Recently, evidence has shown that CHODL is overexpressed in the majority of lung cancers, indicating a possible correlation between CHODL and invasion activity of lung cancer cells (14). IHC staining revealed that short survival of patients with non-small cell lung cancer (NSCLC) was associated with strong CHODL staining, and multivariate analysis confirmed this to be an independent prognostic factor. Induction of exogenous CHODL overexpression conferred growth and invasive activity to mammalian cells, while downregulation of CHODL by small interfering RNAs suppressed growth of lung cancer cells. CHODL is likely to be a prognostic factor and potential target for the development of anticancer drugs (14).

$\mathrm{HCC}$ is prone to rapid progression and metastasis, leaving cytotoxic chemotherapy ineffective, and making it necessary to investigate new diagnostic and therapeutic biomarkers. In this study, we aimed to explore the role of CHODL in HCC migration and invasion, and to evaluate a possible clinical application.

\section{Materials and methods}

Specimens and ethics statement. The use of four human HCC specimens was approved by the Sun Yat-Sen Memorial Hospital, Sun Yat-Sen University (Guangzhou, China). Four human normal liver tissue specimens without hepatitis or cirrhosis were obtained by resection from patients in Sun Yat-Sen Memorial Hospital, Sun Yat-Sen University, between August 2012 and April 2014. All specimens were collected in liquid nitrogen and stored at $-80^{\circ} \mathrm{C}$. Informed written consent was obtained from all participating subjects. All human experiments were conducted with the approval of the Ethics Committee of Sun Yat-Sen Memorial Hospital, Sun Yat-Sen University, and were performed in accordance with the ethical standards of the Helsinki Declaration.

Cell culture. HEK293T cells, the human normal liver cell line L02 and the HCC cell lines HepG2, Hun7, SMMC7721, Bel7402, MHCC-97L and MHCC-97H were purchased from the Type Culture Collection of the Chinese Academy of Sciences (Shanghai, China). Cells were grown in Dulbecco's modified Eagle's medium (Gibco; Thermo Fisher Scientific, Inc., Waltham, MA, USA) supplemented with $10 \%$ defined fetal bovine serum (FBS) (Gibco; Thermo Fisher Scientific, Inc.), $100 \mathrm{U} / \mathrm{ml}$ penicillin, $100 \mu \mathrm{g} / \mathrm{ml}$ streptomycin and $2 \mathrm{mmol} / \mathrm{l}$ glutamine (Thermo Fisher Scientific, Inc.). The cells were cultured in $5 \% \mathrm{CO}_{2}$ humidified at $37^{\circ} \mathrm{C}$.

IHC. Formalin-fixed and paraffin-embedded slides from human HCC specimens and human normal liver tissue specimens were incubated with the specific primary anti-CHODL antibody (1:400; ab76710; Abcam, Cambridge, MA, USA) for $30 \mathrm{~min}$ at $37^{\circ} \mathrm{C}$, and subsequently incubated in a moist chamber shaking overnight at $4^{\circ} \mathrm{C}$. This was followed by three 5-min washes with PBS, and slides were then incubated with the biotin-avidin secondary antibody (1:1,000; ab7235; Abcam) at $37^{\circ} \mathrm{C}$ for $30 \mathrm{~min}$. Slides then were washed with PBS for $5 \mathrm{~min}$ (three times), and incubated with an avidin-biotin-peroxidase complex (1:1,000; Vectastain; Vector Laboratories, Inc.,
Burlingame, CA, USA) at $37^{\circ} \mathrm{C}$ for $30 \mathrm{~min}$. The sections were then stained with 3,3'-diaminobenzidine and counterstained with hematoxylin for $2 \mathrm{~min}$, dehydrated in gradient ethanol, cleared in xylene and cover-slipped with neutral balsam. Negative controls were included in all steps, which were incubated with PBS instead of the specific primary antibodies.

RNA isolation and RT-quantitative $(q) P C R$. Total RNA was extracted using TRIzol Reagent (Invitrogen; Thermo Fisher Scientific, Inc.) according to the manufacturer's instructions, and was subsequently subjected to RT with QuantiTect Reverse Transcription kit (Qiagen, Inc., Valencia, CA, USA). The CHODL and GAPDH sequences were obtained from PubMed GenBank (https://www.ncbi.nlm.nih.gov/nuccore/BC009418.1 and https:/www.ncbi.nlm.nih.gov/nuccore/NM_002046). CHODL expression was analyzed quantitatively using the miScript PCR System (Qiagen, Inc.) on a Stratagene MX3500P (Agilent Technologies, Inc., Santa Clara, CA, USA). The system consists of the miScript Reverse Transcription kit (Qiagen, Inc.) and the miScript SYBR Green PCR kit (Qiagen, Inc.). The housekeeping gene GAPDH was used as an internal control. Gene-specific primers were used: CHODL-forward (F) 5'-GGAAGGAAA GGAACTACGAAATC-3' and CHODL-reverse (R) 5'-GTT AAAAGGAGCACAGGGACATA-3'. Primers for GAPDH were as follows: GAPDH-F: 5'-GAGTCAACGGATTTGGTCGT-3' and GAPDH-R: 5'-GACAAGCTTCCCGTTCTCAG-3'. All primers were synthesized by Thermo Fisher Scientific, Inc. Equal amounts of RNA were reverse transcribed into complementary (c) DNA. The RT reaction was performed at $42^{\circ} \mathrm{C}$ for $30 \mathrm{~min}$ and then $70^{\circ} \mathrm{C}$ for $15 \mathrm{sec}$. qPCR was performed using the cDNA as template under the following conditions: PCR initial activation at $95^{\circ} \mathrm{C}$ for $15 \mathrm{~min}$, followed by 40 cycles at $94^{\circ} \mathrm{C}$ for $15 \mathrm{sec}, 55^{\circ} \mathrm{C}$ for $30 \mathrm{sec}$ and $70^{\circ} \mathrm{C}$ for $30 \mathrm{sec}$. The expression level of CHODL was normalized as relative expression to GAPDH. Relative expression was calculated as $2^{-\Delta \Delta \mathrm{Cq}}$, where $\mathrm{Cq}$ is the quantification cycle and $-\Delta \Delta \mathrm{Cq}=-(\mathrm{Cq}$ CHODL-Cq GADPH). All experiments were performed in triplicate.

Western blot analysis. Human HCC samples and normal liver specimens (100 mg each) were lysed in $1 \mathrm{ml}$ lysis buffer containing $1 \%$ phenylmethanesulfonyl fluoride following the manufacturer's instructions for the radioimmunoprecipitation assay kit (Beyotime Institute of Biotechnology). The lysates were centrifuged at $12,000 \mathrm{rpm}(9,901 \mathrm{x} \mathrm{g})$ for $15 \mathrm{~min}$ at $4^{\circ} \mathrm{C}$ twice to remove cellular debris. Protein concentrations in the supernatant were determined by the bicinchoninic acid assay (Thermo Fisher Scientific, Inc.). An equal amount of protein was separated by $10 \%$ SDS-PAGE, then transferred to polyvinylidene difluoride membranes and blocked with TBS buffer containing $0.5 \%$ Tween 20 (TBST) and 5\% non-fat milk for $1 \mathrm{~h}$ at room temperature. Afterwards, the membranes were incubated with the specific primary antibody against CHODL (1:100; Beyotime Institute of Biotechnology) overnight at $4^{\circ} \mathrm{C}$. After three 7-min washes with TBST, the blots were incubated with a horseradish peroxidase-labeled secondary antibody $(1: 1,000 ;$ ab181658; Abcam) for $1 \mathrm{~h}$ at room temperature. The presence of peroxidase was detected with an enhanced chemiluminescence reagent (Shanghai Long Island Biotech Co., Ltd.) following three 10-min washes with TBST. Blots were normalized to GAPDH after background subtraction. The 
anti-GAPDH antibody (ab37168) was obtained from Beyotime Institute of Biotechnology and was diluted 1:100. Each experiment was repeated in triplicate with similar results.

Construction of lentiviral vectors and generation of stable cell lines. Total RNA was extracted and subsequently subjected to $\mathrm{RT}$ as mentioned above. The RT reaction was performed at $70^{\circ} \mathrm{C}$ for $5 \mathrm{~min}$ and then $37^{\circ} \mathrm{C}$ for $30 \mathrm{~min}$. A third generation lentiviral vector pCDH-CMV-EF1-copGFP (Invitrogen; Thermo Fisher Scientific, Inc.) was used to overexpress CHODL following the manufacturer's instructions. The oligonucleotides encoding the designed CHODL sequence (dihydropyrimidinase like 3) were synthesized by Thermo Fisher Scientific, Inc. and inserted into the restriction sites $X b a \mathrm{I}$ and $E c o$ RI of the multiple cloning site of the vector. Infectious lentiviral vectors were harvested at $48 \mathrm{~h}$ post-transfection, and virus titer was calculated by fluorescence-activated cell sorting analysis of green fluorescent protein (GFP)-positive HEK293T cells. The titers were then adjusted to $10^{9}$ transducing units $/ \mathrm{ml}$. Correct CHODL insertions were confirmed by direct DNA sequencing. HEK293T cells were then co-transfected with the recombinant replication competent virions (pCDH-CMV-hCHODL-EF1-copGFP) and three helper plasmids (pGag/Pol, pRev and pVSV-G)(Shanghai GenePharma Co., Ltd., Shanghai, China) using Lipofectamine 2000 (Invitrogen; Thermo Fisher Scientific, Inc.) according to the manufacturer's protocol. SMMC7721 cells were seeded on day 0 and the medium was removed on day 1 . Then, SMMC7721 cells were transduced with the infectious lentiviruses in fresh transduction medium supplemented with Polybrene ( $8 \mu \mathrm{g} / \mathrm{ml}$; Sigma-Aldrich; Merck Millipore, Darmstadt, Germany). At $24 \mathrm{~h}$ post-infection, cells were treated with fresh medium for $6 \mathrm{~h}$ and then cultured in complete medium containing puromycin $(2 \mu \mathrm{g} / \mathrm{ml})$ for $10-12$ days before being used for experiments. The percentage of GFP-expressing cells and the fluorescence intensity were observed under a fluorescence microscope (Olympus Corporation, Tokyo, Japan). CHODL expression was determined using both RT-qPCR and western blotting post-transduction, as aforementioned.

Flow cytometry (FCM). Cell cycle and apoptosis analyses were performed using propidium iodide (PI) and fluorescein isothiocyanate (FITC)-Annexin V staining (Bio-Rad Laboratories, Inc., Hercules, CA, USA). For cell cycle investigation, the human HCC cell line SMMC7721 and the normal liver cell line L02 were seeded in 96-well plates at $1 \times 10^{4}$ cells per well. At 24, 48 and $72 \mathrm{~h}$ after transduction, the cells were lysed with TRIzol for $30 \mathrm{~min}$ in an ice bath, and then trypsinized (Invitrogen; Thermo Fisher Scientific, Inc.) and washed twice with precooled PBS. Cells were centrifuged at 3,000 rpm $(619 \times \mathrm{x})$ for $5 \mathrm{~min}$ at $4^{\circ} \mathrm{C}$ and fixed in $70 \%$ precooled ethanol for $24 \mathrm{~h}$ at $4^{\circ} \mathrm{C}$. A total of $1 \times 10^{5}$ cells were stained with PI for $10 \mathrm{~min}$. The cell cycle distribution was analyzed by FCM (FACSCalibur; BD Biosciences, San Jose, CA, USA). For the apoptosis analysis, $1 \times 10^{5}$ cells were trypsinized (Invitrogen; Thermo Fisher Scientific, Inc.) and washed with precooled PBS. Cells were collected by centrifugation at 3,000 rpm $(619 \mathrm{x} \mathrm{g})$ for $5 \mathrm{~min}$ at $4^{\circ} \mathrm{C}$ and fixed with $75 \%$ precooled alcohol. The cells were then incubated with FITC-Annexin V and PI for 5 min and analyzed by FCM. Each experiment was repeated in triplicate with similar results.
Cell proliferation assay. A total of $1 \times 10^{4}$ post-transfection SMMC7721 cells were seeded into 96-well plates and cultured overnight at $5 \% \mathrm{CO}_{2}$ humidified at $37^{\circ} \mathrm{C}$. Once the cells were completely adherent to the wells, at 20,44 and $68 \mathrm{~h}, 20 \mu \mathrm{l}$ $5 \mathrm{mg} / \mathrm{ml}$ MTT (Sigma-Aldrich; Merck Millipore) was added to the plates, and the cells were cultured for an additional $4 \mathrm{~h}$. Culture medium was then removed, and $150 \mu \mathrm{l}$ dimethyl sulfoxide was added to the plates, followed by a 15 -min incubation. The absorbance at $490 \mathrm{~nm}$ was measured to determine the number of viable cells in each well. All experiments were performed in triplicate.

Cell Matrigel migration and invasion assays. For the invasion assays, Transwell upper chambers (Costar; Corning Incorporated, Corning, NY, USA) $(8 \mu \mathrm{m})$ were incubated with Matrigel (BD Biosciences) $(2.5 \mathrm{mg} / \mathrm{ml}$, diluted in serum-deprived medium) for $5 \mathrm{~h}$ at $37^{\circ} \mathrm{C}$. In total, $1.0 \times 10^{5} \mathrm{SMMC} 772$ cells were seeded in the upper Transwell chambers, and $500 \mu 1$ substrate containing 10\% FBS was added into the lower chamber. Cells were incubated for $24 \mathrm{~h}$, washed twice with PBS and fixed with $4 \%$ paraformaldehyde. Finally, the insert membranes were stained with crystal violet. Ten fields were randomly chosen, and the permeating cells were counted for each membrane under an inverted microscope (Leica Microsystems GmbH, Wetzlar, Germany). For the migration assay, the insert membranes were not coated with Matrigel, and the cells were cultured under the same conditions. Each experiment was repeated in triplicate with similar results.

Statistical analysis. Data are expressed as means \pm standard deviation, and the Student's $t$ test was used to compare the two groups. One-way analysis of variance was used when comparing multiple groups, followed by the least significant difference $t$ test to look for differences amongst groups. Statistical analyses were performed using SPSS v19.0 (SPSS, Inc., Chicago, IL, USA). $\mathrm{P}<0.05$ was considered to indicate a statistically significant difference.

\section{Results}

CHODL is significantly downregulated in HCC clinical samples and cell lines. RT-qPCR analyis showed that CHODL mRNA was significantly lower in the HCC clinical samples than in the normal liver tissues $(\mathrm{P}<0.01)$ (Fig. 1A). Similar results were obtained in HCC cell lines. CHODL mRNA expression was downregulated in the HCC cell lines HepG2, Hun7, SMMC7721, Bel7402, MHCC-97L and MHCC-97H compared with the human normal liver cell line L02 $(\mathrm{P}<0.001)$ (Fig. 1B). The expression of CHODL was lowest in SMMC7721 cells (Fig. 1B). Therefore, SMMC7721 cells were selected for subsequent analysis to elucidate the possible association between CHODL and liver metastasis.

A western blot assay was used to detect protein expression of CHODL. The results showed that CHODL expression in all four HCC clinical samples was lower than in the normal liver tissues (Fig. 2A). IHC staining was performed to investigate the expression of CHODL in the four HCC clinical samples. In accord with the western blot results, CHODL staining in human normal liver tissues was positive (uniformly yellow), but was negative in HCC samples (Fig. 2B). 
A

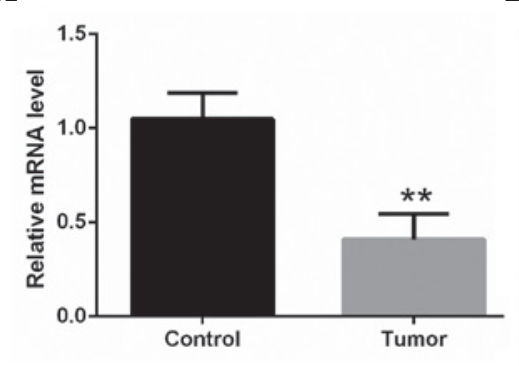

B

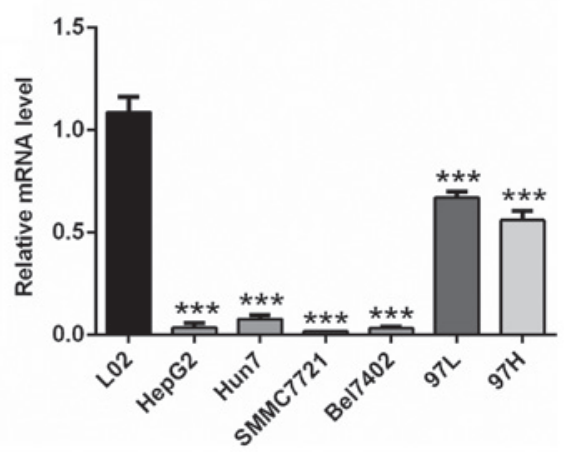

Figure 1. mRNA expression of CHODL in HCC and normal human liver tissue. (A) RT-qPCR analyses of CHODL mRNA expression in HCC clinical samples and normal human liver tissue. (B) RT-qPCR analyses of CHODL mRNA expression in HCC cell lines. Relative mRNA expression was quantified as $2^{-\Delta \Delta C q}$, GAPDH was used as an internal control. All experiments were performed at least in triplicate. ${ }^{* *} \mathrm{P}<0.01,{ }^{* * *} \mathrm{P}<0.001$. mRNA, messenger RNA; RT-qPCR, reverse transcription-quantitative polymerase chain reaction; HCC, hepatocellular carcinoma; CHODL, chondrolectin.

A

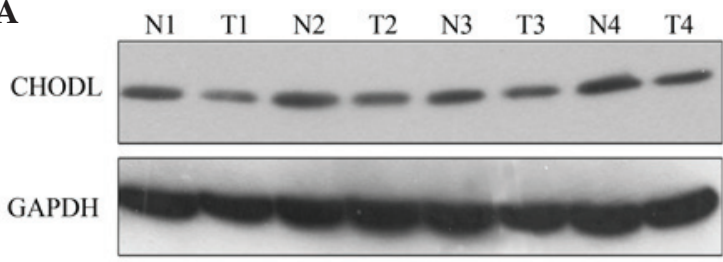

B
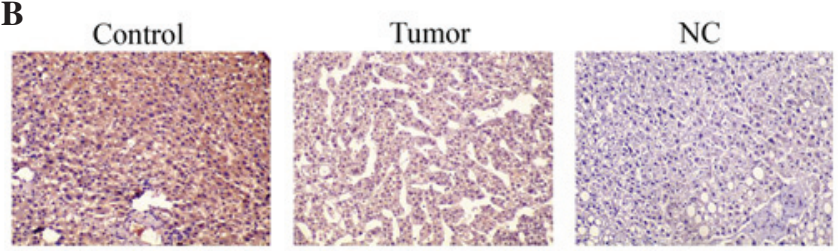

Figure 2. Western blot analysis and IHC of the expression of CHODL in HCC and normal human liver tissue. (A) Western blotting of CHODL protein expression in HCC clinical samples and normal liver tissues revealed significantly lower protein expression in four HCC clinical samples compared with four normal liver tissues. (B) A representative IHC staining of normal liver tissues and HCC clinical samples: Human normal liver tissue was positive (uniformly yellow), while HCC sample was negative (original magnification, x400; scale bar, $50 \mu \mathrm{m}$ ). N, normal; T, tumor; HCC, hepatocellular carcinoma; CHODL, chondrolectin; IHC, immunohistochemistry.

Lentiviral vector mediates overexpression of CHODL in SMMC7721 cells. SMMC7721 cells were transduced with the recombinant lentiviral CHODL overexpression vector or with empty vector. RT-qPCR analysis was performed to quantify the upregulation of CHODL mRNA levels in SMMC7721 cells. CHODL mRNA expression was increased by $\sim 5$-fold over the control $(\mathrm{P}<0.01)$ (Fig. 3A). Western blotting confirmed CHODL overexpression in SMMC7721 cells. The protein expression level of CHODL in the overexpression group was significantly upregulated compared with that in the mock vector group (Fig. 3B). These results showed that CHODL expression was increased by the lentiviral vectors.

Overexpression of CHODL increases migration and invasion in SMMC7721 cells. MTT assays and FCM results demonstrated that cell metabolism (Fig. 4A) and apoptosis (Fig. 4B) were not significantly different between CHODL-overexpressing cells and control cells. However, the CHODL overexpression group displayed more cells in the $\mathrm{G} 2 / \mathrm{M}$ phase than the control group (Fig. 4C).
A

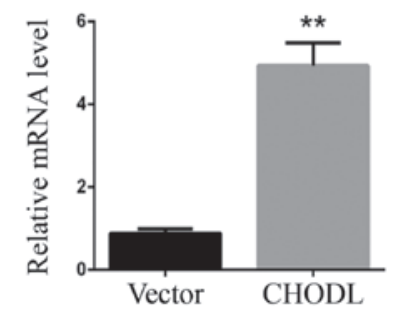

B

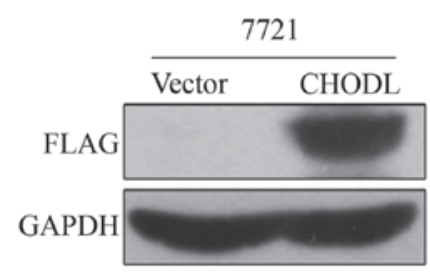

Figure 3. Establishment of a CHODL-overexpressing cell line. (A) Reverse transcription-quantitative polymerase chain reaction results indicated higher mRNA expression of CHODL in transduced SMMC7721 cells than in the negative control cells (transduced with mock vector). ${ }^{* *} \mathrm{P}<0.01$. (B) Western blot analysis of CHODL protein expression in transduced SMMC7721 cells indicated that these cells displayed higher CHODL protein expression than cells transduced with mock vector. Vector, mock vector; CHODL, CHODL group containing CHODL overexpression vector. All experiments were performed at least in triplicate. CHODL, chondrolectin; mRNA, messenger RNA.

To determine whether upregulation of CHODL was correlated to HCC migration, we used Transwell assay to determine the ability of cell migration and invasion. As shown in Fig. 5A, increased migrating cell numbers were observed in the CHODL-overexpressing SMMC7221 cells, indicating a possible association between CHODL and HCC migration $(\mathrm{P}<0.01)$ (Fig. 5A). To further elucidate the effect of CHODL on HCC cell invasion, cellular invasion was examined via Matrigel assay. The upregulation of CHODL in SMMC7721 cells significantly enhanced the cells invasive activity (Fig. 5B). These results suggested a contribution of CHODL to the high risk of malignant metastasis in HCC.

\section{Discussion}

HCC is the fifth most common cancer in men and the seventh in women (15). Liver cirrhosis and/or chronic viral hepatitis have been proposed as a predisposing factor for hepatocarcinogenesis $(1,6)$. Chronic infection with hepatitis B virus (HBV) represents a major risk factor for HCC in Southeast Asia and Africa, while lingering infection with hepatitis $C$ virus is the predominant risk factor for $\mathrm{HCC}$ in Western countries and Japan $(2,16,17)$. At the asymptomatic early stages of HCC, 
A

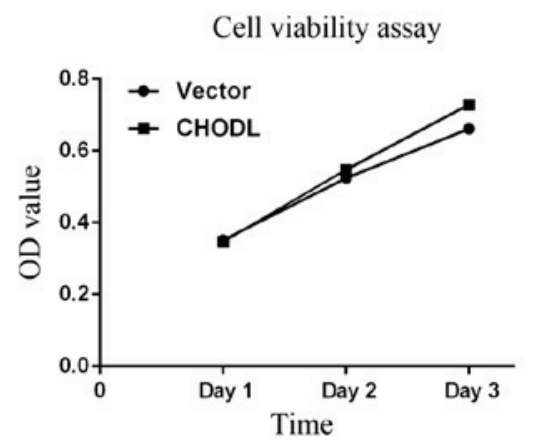

B
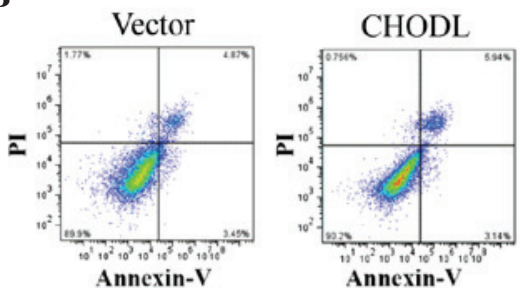

C

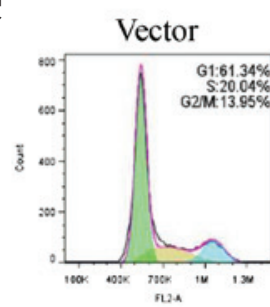

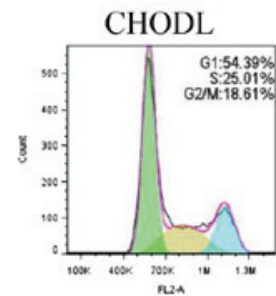
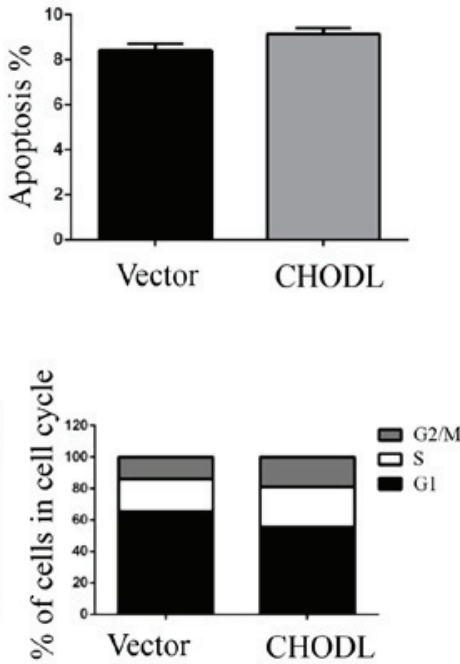

Figure 4. Cell cycle, proliferation rate and apoptosis in cell lines with CHODL overexpression. (A) Cell viability was evaluated by MTT assay at each indicated time point. (B) Investigation of cell cycle status by FCM with PI staining. (C) Apoptosis in cancer cells was analyzed by FCM with Annexin-V/PI. FCM results revealed that cell cycle and apoptosis were unaffected by CHODL overexpression. Vector, mock vector; CHODL, CHODL group overexpressing CHODL. The data represent the mean \pm standard deviation of three different experiments. FCM, flow cytometry; CHODL, chondrolectin; OD, optical density; PI, propidium iodide.

A
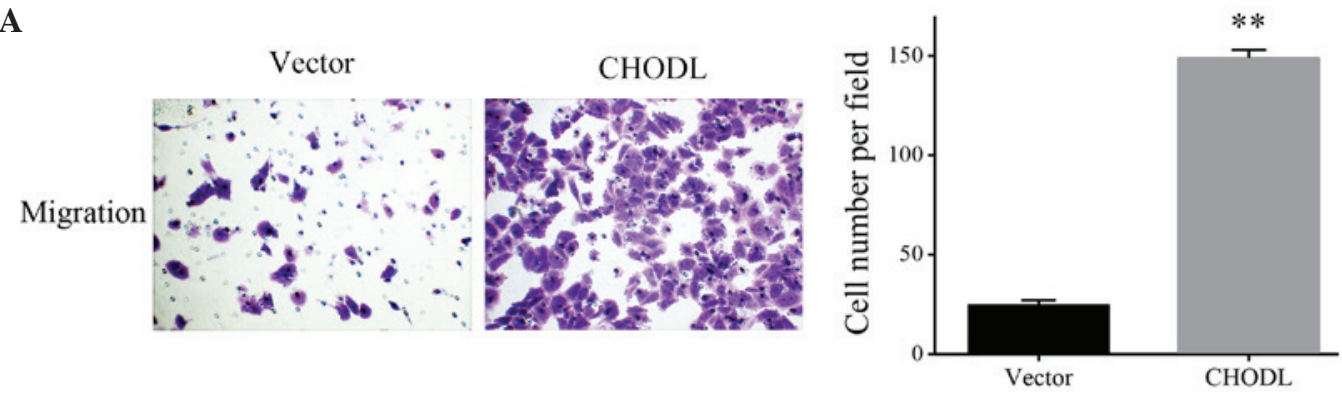

$\mathbf{B}$
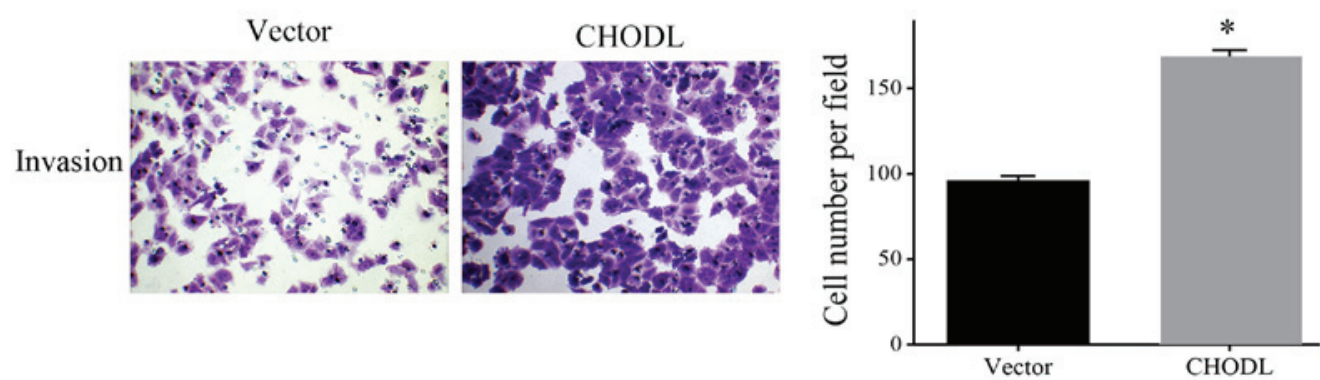

Figure 5. CHODL expression enhances migration and invasiveness. (A) Increase in cell migration of CHODL-overexpressing SMMC7721 cells (magnification, x200; staining, crystal violet). (B) Enhanced invasiveness of SMMC7721 cells in a Matrigel matrix after transduction with a lentiviral vector overexpressing human CHODL. The migration and invasion of cancer cells was measured by Transwell assay. Vector, mock vector; CHODL, CHODL group containing CHODL overexpression vector. Assays were done three times and in triplicate wells. ${ }^{* *} \mathrm{P}<0.01,{ }^{*} \mathrm{P}<0.05$. CHODL, chondrolectin.

ablative therapies, surgical resection or liver transplantation are the first-line treatment $(18,19)$. However, treatment options are limited for most advanced stage patients due to HCC intra-hepatic expansion and/or poor liverfunction (20).

Chronic hepatocyte injury, including continuous inflammatory and oxidative stress, may cause regenerative stimuli in hepatic cells $(5,17)$. This kind of cellular proliferation may lead to mutations in genes controlling proliferation and/or apoptosis, thus leading to hepatic tumorigenesis $(6,17)$. HCC is a chemoresistant tumor, and conventional pharmacological chemotherapy has limited efficacy in clinical benefits and/or prolonged survival $(1,17)$. Therefore, it is imperative to investigate HCC biomarkers related to cell proliferation and migration, which may help to provide curative options for HCC prevention and intervention.

The human CHODL protein belongs to the C-type lectin family and contains an $\mathrm{N}$-terminal signal sequence, a single CRD, a transmembrane region and an intracellular C terminus (8-10). Animal lectin CRDs have been found in many kinds of proteins, including selectin, mannose-binding protein, proteoglycan core protein, lymphocyte immunoglobulin E receptor and hepatic asialoglycoprotein receptor (10). 
The C-type CRDs provide calcium-dependent sugar-binding activity and are involved in many processes, including cell recognition and communication, cell-cell adhesion, and ECM-cell interactions, steps that are involved in and crucial for tumor cell proliferation, migration and invasion $(9,14)$. The full-length CHODL protein is expressed in various human tissues and co-localizes with rBet1, a transmembrane protein that mediates protein transport between the endoplasmic reticulum (ER) and the Golgi apparatus, indicating an important role for CHODL in protein processing (10).

Masuda et al showed that CHODL was increased in NCSLC and was associated with poor prognosis in patients (14). CHODL protein was mainly co-localized with calreticulin (an ER marker) and was expressed in the majority of NSCLC tissues, with the expression of the CHODL transcript being upregulated 5-fold or greater in $63 \%$ of NSCLCs compared with normal lung tissue. Transfection of exogenous CHODL into the lung cancer cell line LC319 significantly enhanced cell growth and invasion, indicating that CHODL was associated with lung oncogenesis and tumor progression (14).

Our study showed that CHODL was significantly decreased in HCC clinical samples compared with normal liver tissue at both the mRNA and protein levels. Similarly, CHODL downregulation was also observed in HCC cell lines in vitro. Lentiviral overexpression assays for CHODL revealed that CHODL could improve HCC cell line SMMC7721 migration and invasion in vitro, but did not affect HCC proliferation. These data suggested a possible contribution of CHODL to the high risk of malignant metastasis in HCC.

This result was quite different from previous data in lung cancer (14). In general, CHODL expression in normal human tissues is very low $(8,13,14)$. In the SMA mouse spinal cord, CHODL-001 isoform expression was reduced before disease onset, and the reduced expression of CHODL-001 in mice survival motor neuron-depleted NSC-34 cells improved neurite outgrowth (11). Adult liver cells retain a remarkable capacity to proliferate in response to liver injury, and can re-enter the cell cycle and divide once or twice before returning to a state of quiescence (17). Hepatic nodules in patients with chronic liver diseases are subdivided into regenerative nodules, low-grade dysplastic nodules, high-grade dysplastic nodules, well-differentiated HCC, moderately-differentiated HCC and poorly-differentiated HCC, showing an ascending order of histological grades and representing a sequence of multistep hepatocarcinogenesis (17). Our study showed that CHODL overexpression led to a higher proportion of cells in the G2/M phase than the control group, which may explain the lower CHODL expression in HCC tissues and in HCC cell lines as compared with the normal liver tissues and cell line (L02), respectively.

Efficient and long-term gene transfer is essential for clinical gene therapy, of which intensity and duration of transgenic expression are the two main parameters (21). Chemically synthesized small hairpin RNAs delivered to target cells are known to be transient, lasting only 3-4 days, with low transfection efficiency $(16,22)$. Lentiviral vectors can achieve $70 \%$ gene transfer efficacy in both dividing and nondividing hepatocarcinoma cells over a 16-h transduction period with a multiplicity of infection of $1(4,21,23)$. It is noteworthy that lentiviral vectors can efficiently transduce HCC cells in the context of HBV infection, which makes gene therapy protocols in combination with other therapeutic approaches (surgery and/or chemotherapy) feasible $(16,22)$. Although there are biosafety concerns, including potential insertional mutagenesis and vector-related toxic side effects to the liver, the current third generation human immunodeficiency virus-1-based lentiviral vectors have minimized the potential risk $(16,24)$.

In summary, while the exact molecular mechanisms behind CHODL involvement in HCC tumorigenesis remain to be studied, our investigation suggests that CHODL plays a role in proliferation and aggression of HCC. CHODL may be a candidate therapeutic and prognostic biomarker for liver cancer.

In this study, we found that the expression of CHODL was significantly lower in HCC clinical samples and in HCC cell lines than in normal liver tissue. Lentivirus-mediated overexpression of CHODL in SMMC7721 liver cancer cells significantly increased cell migration and invasion. Our study demonstrates for the first time a positive correlation between CHODL and HCC metastasis.

\section{Acknowledgements}

The present study was supported by the National Natural Science Foundation of China (Beijing, China; grant no. 81372562) and the Medical Scientific Research Foundation of Guangdong Province, China (grant no. A2015462)

\section{References}

1. Ferrin G, Aguilar-Melero P, Rodríguez-Perálvarez M, Montero-Âlvarez JL and de la Mata M: Biomarkers for hepatocellular carcinoma: Diagnostic and therapeutic utility. Hepat Med 7: 1-10, 2015.

2. Llovet JM, Di Bisceglie AM, Bruix J, Kramer BS, Lencioni R, Zhu AX, Sherman M, Schwartz M, Lotze M, Talwalkar J, et al: Design and endpoints of clinical trials in hepatocellular carcinoma. J Natl Cancer Inst 100: 698-711, 2008.

3. Hong YP, Li ZD, Prasoon P and Zhang Q: Immunotherapy for hepatocellular carcinoma: From basic research to clinical use. World J Hepatol 7: 980-992, 2015.

4. Gerolami R, Uch R, Jordier F, Chapel S, Bagnis C, Bréchot C and Mannoni P: Gene transfer to hepatocellular carcinoma: Transduction efficacy and transgene expression kinetics by using retroviral and lentiviral vectors. Cancer Gene Ther 7: 1286-1292, 2000.

5. Pez F, Lopez A, Kim M, Chapel S, Bagnis C, Bréchot C and Mannoni P: Wnt signaling and hepatocarcinogenesis: Molecular targets for the development of innovative anticancer drugs. J Hepatol 59: 1107-1117, 2013.

6. Bhatia D, Thoppil RJ, Mandal A, Samtani KA, Darvesh AS and Bishayee A: Pomegranate bioactive constituents suppress cell proliferation and induce apoptosis in an experimental model of hepatocellular carcinoma: Role of Wnt/ $\beta$-catenin signaling pathway. Evid Based Complement Alternat Med 2013: 371813, 2013.

7. Manu KA, Shanmugam MK, Ong TH, Subramaniam A, Siveen KS, Perumal E, Samy RP, Bist P, Lim LH, Kumar AP, et al: Emodin suppresses migration and invasion through the modulation of CXCR4 expression in an orthotopic model of human hepatocellular carcinoma. PLoS One 8: e57015, 2013.

8. Weng L, Smits P, Wauters J and Merregaert J: Molecular cloning and characterization of human chondrolectin, a novel type I transmembrane protein homologous to C-type lectins. Genomics 80: 62-70, 2002.

9. Weng L, Hubner R, Claessens A, Smits P, Wauters J, Tylzanowski P, Van Marck E and Merregaert J: Isolation and characterization of chondrolectin (Chodl), a novel C-type lectin predominantly expressed in muscle cells. Gene 308: 21-29, 2003. 
10. Weng L, Van Bockstaele DR, Wauters J, Van Marck E, Plum J, Berneman ZN and Merregaert J: A novel alternative spliced chondrolectin isoform lacking the transmembrane domain is expressed during $\mathrm{T}$ cell maturation. J Biol Chem 278: 19164-19170, 2003.

11. Sleigh JN, Barreiro-Iglesias A, Oliver PL, Biba A, Becker T, Davies KE, Becker CG and Talbot K: Chondrolectin affects cell survival and neuronal outgrowth in in vitro and in vivo models of spinal muscular atrophy. Hum Mol Genet 23: 855-869, 2014.

12. Zhong Z, Ohnmacht J, Reimer MM, Bach I, Becker T and Becker CG: Chondrolectin mediates growth cone interactions of motor axons with an intermediate target. J Neurosci 32: 4426-4439, 2012.

13. Claessens A, Van de Vijver K, Van Bockstaele DR, Wauters J, Berneman ZN, Van Marck E and Merregaert J: Expression and localization of CHODLDeltaE/CHODLfDeltaE, the soluble isoform of chondrolectin. Cell Biol Int 31: 1323-1330, 2007.

14. Masuda K, Takano A, Oshita H, Akiyama H, Tsuchiya E, Kohno N, Nakamura Y and Daigo Y: Chondrolectin is a novel diagnostic biomarker and a therapeutic target for lung cancer. Clin Cancer Res 17: 7712-7722, 2011

15. Dang H, Steinway SN, Ding W and Rountree CB: Induction of tumor initiation is dependent on CD44s in c-Met+ hepatocellular carcinoma. Bmc Cancer 15: 161, 2015.

16. Deng L, Li G, Xi L, Yin A, Gao Y, You W, Wang X and Sun B: Hepatitis B virus inhibition in mice by lentiviral vector mediated short hairpin RNA. BMC Gastroenterol 9: 73, 2009.

17. Marra M, Sordelli IM, Lombardi A, Lamberti M, Tarantino L, Giudice A, Stiuso P, Abbruzzese A,Sperlongano R, Accardo M, et al: Molecular targets and oxidative stress biomarkers in hepatocellular carcinoma: An overview. J Transl Med 9: 171, 2011.
18. Altimari A, Fiorentino M, Gabusi E, Gruppioni E, Corti B, D'Errico A and Grigioni WF: Investigation of ErbB1 and ErbB2 expression for therapeutic targeting in primary liver tumours. Dig Liver Dis 35: 332-338, 2003.

19. Forner A, Hessheimer AJ, Isabel Real M and Bruix J: Treatment of hepatocellular carcinoma. Crit Rev Oncol Hematol 60: 89-98, 2006.

20. Fan ST, Lo CM, Liu CL, Lam CM, Yuen WK, Yeung C and Wong J: Hepatectomy for hepatocellular carcinoma: Toward zero hospital deaths. Ann Surg 229: 322-330, 1999.

21. Chang LJ, Urlacher V, Iwakuma T, Cui Y and Zucali J: Efficacy and safety analyses of a recombinant human immunodeficiency virus type 1 derived vector system. Gene Ther 6: 715-728, 1999.

22. Liu Y, Butterfield LH, Fu X, Song Z, Zhang X, Lu C, Ding G and $\mathrm{Wu} \mathrm{M}$ : Lentivirally engineered dendritic cells activate AFP-specific T cells which inhibit hepatocellular carcinoma growth in vitro and in vivo. Int J Oncol 39: 245-253, 2011.

23. Wang Q, Liu QY, Liu ZS, Qian Q, Sun Q and Pan DY: Lentivirus mediated shRNA interference targeting MAT2B induces growth-inhibition and apoptosis in hepatocelluar carcinoma. World J Gastroenterol 14: 4633-4642, 2008.

24. Kafri T, Blomer U, Peterson DA, Gage FH and Verma IM: Sustained expression of genes delivered directly into liver and muscle by lentiviral vectors. Nat Genet 17: 314-317, 1997. 